\title{
A Nomogram for Predicting Mammaprint Results in Women with T1-3N0-1M0 Hormone Receptor- Positive and Human Epidermal Growth Factor Receptor-2-Negative Breast Cancer
}

\section{Young Joo Lee}

Department of Surgery, St Mary's Hospital, The Catholic University of Korea, College of Medicine

Young Sol Hwang

University of Ulsan College of Medicine

Junetae Kim

Graduate School of Cancer Science and Policy, National Cancer Center, Goyang-si, Republic of Korea Sei-Hyun Ahn

Division of Breast Surgery, Department of Surgery, University of Ulsan College of Medicine, Asan Medical Center, Seoul, Republic of Korea

\section{Byung Ho Son}

Division of Breast Surgery, Department of Surgery, University of Ulsan College of Medicine, Asan Medical Center, Seoul, Republic of Korea

\section{Hee Jeong Kim}

Division of Breast Surgery, Department of Surgery, University of Ulsan College of Medicine, Asan Medical Center, Seoul, Republic of Korea

\section{Beom Seok Ko}

Division of Breast Surgery, Department of Surgery, University of Ulsan College of Medicine, Asan Medical Center, Seoul, Republic of Korea

Jisun Kim

Division of Breast Surgery, Department of Surgery, University of Ulsan College of Medicine, Asan Medical Center, Seoul, Republic of Korea

\section{Yong Chung}

Division of Breast Surgery, Department of Surgery, University of Ulsan College of Medicine, Asan Medical Center, Seoul, Republic of Korea

\section{Jong Won Lee}

Division of Breast Surgery, Department of Surgery, University of Ulsan College of Medicine, Asan Medical Center, Seoul, Republic of Korea

\section{Sae Byul Lee ( $\nabla$ newstar153@hanmail.net)}

Division of Breast Surgery, Department of Surgery, University of Ulsan College of Medicine, Asan Medical Center, Seoul, Republic of Korea 


\section{Research Article}

Keywords: breast cancer, MammaPrint, nomogram, prognosis

Posted Date: August 13th, 2021

DOl: https://doi.org/10.21203/rs.3.rs-764423/v1

License: (c) (i) This work is licensed under a Creative Commons Attribution 4.0 International License. Read Full License 


\section{Abstract}

\section{Purpose}

We aimed to develop a prediction MammaPrint (MMP) genomic risk assessment nomogram model for hormone-receptor-positive and human epidermal growth factor receptor-2 (HER2)-negative breast cancer and minimal axillary burden (N0-1) tumors using clinicopathological factors of patients who underwent an MMP test for decision making regarding adjuvant chemotherapy.

\section{Methods}

A total of 409 T1-3 N0-1 M0 hormone receptor-positive and HER2-negative breast cancer patients whose MMP genomic risk results were available at Asan Medical Center from 2017 to 2020 were enrolled. Patients were randomly assigned to training and validation subsets and logistic regression was performed.

\section{Results}

The primary cohort $(n=409)$ included $216(53.1 \%)$ T2-3 and $388(94.8 \%)$ N1 patients. No patients were estrogen-receptor-negative or -weak, 175 (42.7\%) had a high proliferation index (Ki-67 $\geq 20 \%$ ), and 225 (55.0\%) were premenopausal. Multivariate analysis revealed that the age at diagnosis, progesterone receptor (PR) score, nuclear grade, and Ki-67 were significantly associated with MMP risk results. We developed an MMP low-risk predictive nomogram. The area under the receiver operating characteristic curve was 0.82 ( $95 \%$ confidence interval $[\mathrm{Cl}], 0.77$ to 0.87 ). When applied to the validation group, the nomogram was accurate with an area under the curve of $0.77(95 \% \mathrm{Cl}, 0.68$ to 0.86$)$.

\section{Conclusion}

Our nomogram, which incorporates four traditional prognostic factors, i.e., age, PR, nuclear grade, and Ki67 , could predict the probability of obtaining a low MMP risk in a cohort of intermediate clinical risk patients. This nomogram can aid the selection of patients who need additional MMP testing.

\section{Introduction}

The decision as to whether administer adjuvant chemotherapy for hormone receptor-positive and human epidermal growth factor receptor 2 (HER2)-negative breast cancer is becoming increasingly complex. In patients who are positive for hormone receptor and negative for HER2, this decision is made by predicting the prognosis using genomic analysis of individual tumor cells, besides considering the previously known prognostic factors [1-4]. Cancer treatment guidelines recommend the use of genomic tests such as Oncotype DX for analyzing how a set of genes can affect cancer prognosis; the results of these tests enable decision making regarding the use adjuvant chemotherapy for patients without lymph node metastasis [5-7]. In addition to Oncotype DX, MammaPrint (MMP), a 70-gene-signature-based test is recommended for patients with minimal nodal involvement (N0-1)[8]. Until we obtain stronger evidence 
for the use of Oncotype DX in patients with axillary node metastasis (RxPONDER) [9], MMP is recommended for making a decision regarding the use of adjuvant chemotherapy with the highest evidence level for hormone receptor positive, HER2 negative, and node positive breast cancer irrespective of menopausal status [10].

Nowadays, genomic assays play an important role in decision making regarding adjuvant treatment and their use has become a standard procedure. However, these also have some disadvantages. Recent reports have revealed a long-term survival benefit in premenopausal women with intermediate Recurrence Score or in a clinical high/genomic low risk group treated with adjuvant chemotherapy $[5,11]$. Thus, by not considering the clinical factors and instead using individual cancer genes, the tools developed to simplify cancer treatment are now leading us back to the era when clinicopathological factors were important. Additionally, the cost of genomic assays can be a burden for patients who are not covered by medical insurance. Therefore, we had created a nomogram for predicting Oncotype DX Recurrence Score 3 years ago [12], and initiated an effort to provide clinicians with an easier decision making process regarding adjuvant chemotherapy, thereby aiding the treatment of patients who are not able to afford the expenditure associated with genomic tests.

The purpose of this study is to develop a nomogram model for MMP genomic risk assessment in patients with hormone receptor positive, HER2 negative, and minimal axillary burden (N0-1) breast cancer by using widely used clinicopathological factors for predicting breast cancer outcomes in a subset of patients subjected to the MMP test to enable decision making regarding adjuvant chemotherapy.

\section{Materials And Methods}

\section{Patient selection and pathology variable selection}

The primary cohort was selected through the evaluation of the initial record of all T1-3N0-1M0 hormone receptor-positive and HER2-negative breast cancer patients whose tumor tissues were analyzed using the MMP test between 2017 and 2020 at Asan Medical Center, Seoul, Republic of Korea. A study data set of 409 cases with available MMP test results was used to build the prediction models. A total of 312 patients were selected for the training group and 97 patients were selected for the validation group in the primary cohort. The clinical data of the patients were obtained from the electronic medical records. Clinical information, including patient age, tumor size, lymph node (LN) status, pathological stage, histological grade, nuclear grade, lymphovascular invasion (LVI), Ki-67, p53, and molecular subtypes according to the ER, PR, and HER2 status based on immunohistochemistry or fluorescence in situ hybridization were obtained. Immunohistochemistry for ER, PR, HER2, and Ki-67 and in situ hybridization for HER2 were performed at the Asan Medical Center pathology laboratories.

\section{Statistical analysis}

MMP test results were categorized as low or high recurrence risk. Chi-square test and Fisher's exact test were used to compare the MMP results among clinicopathological characteristics. Initial variable 
selection was performed on the basis of univariate linear regression in development samples. Stepwise multiple logistic regression analysis was used to develop a predictive model for a low risk of MMP results. Four factors, including age at diagnosis (20-100), nuclear grade (range, 1-3), Allred scores for PR status (range, $0-8)$ and Ki-67 labeling index (0-100) were found to contribute significantly by the multivariate logistic regression model. We conducted a robustness analysis to validate our model by employing a random sampling validation procedure. We randomly partitioned the data into two subsets where the sample size was 312:97 at a ratio of 7:3, ran each random effect logistic regression using the training and test sets, employed the receiver operating characteristic curve analysis, and calculated the area under the curve (AUC). All data analyses were performed using R statistical package ver. 3.2.0 (http://r-project.org). Significance level was set at 0.05 and all p-values were two-sided.

\section{Ethical statement}

The project was performed in accoardance with the Declaration of Helsinki and the relevant guidelines and regulation, reviewed and approved by the Asan Medical Center institutional review board (20200037). Due to the retrospective nature of the study, the requirement for informed consent was waived and approved by Asan Medical Center institutional review board.

\section{Results}

\section{Baseline characteristics}

Data from patients $(n=409)$ whose tumor tissues were analyzed using the MMP test were used to develop the nomogram for predicting patients with a low risk of MMP. A detailed comparison of the clinical characteristics of all patients based on the MMP results is shown in Table 1. The average age at diagnosis was $53.3 \pm 9.3$ years in the MMP low-risk group vs. $47.9 \pm 9.8$ years in the MMP high-risk group $(p<0.001)$ and the average tumor size was $2.2 \pm 1.1 \mathrm{~cm}$ vs. $2.4 \pm 1.0 \mathrm{~cm}, p=0.018$. In the MMP high-risk group, a significantly higher rate of histological and nuclear grade and a higher Ki-67 level ( $\geq 20 \%)$, all $p<$ 0.001 , were observed. With respect to the receptor status, most of the patients had a strong Allred score (7-8) of ER. In the MMP high-risk group, 6 patients (3.6\%) had an intermediate ER score (5-6) compared with $2(0.8 \%)$, in the low-risk group. None of the patients had a negative $(0-2)$ or weak positive (3-4) ER score. MMP low-risk group had a better strong PR status (7-8) rate (68.8\%) compared with the MMP lowrisk group $(53.3 \%)$ and a lower rate of negative or weak PR status, $p<0.001$. No difference was seen in the surgical methods. More T2 tumors in the high-risk group $(46.2 \%$ vs. $58.7 \%, p=0.045)$ without any significant difference in the pathological $\mathrm{N}$ stage and final stage were observed. There was no significant difference in the number of positive nodes, $p=0.942$; however, MMP high-risk group had a slightly larger size of positive nodes $(5.4 \pm 3.9 \mathrm{~mm}$ vs. $6.3 \pm 4.8 \mathrm{~mm}, \mathrm{p}=0.042)$. More women in the high-risk group were premenopausal $(66.9 \%, p<0.001)$.

\section{Development of model predicting MMP results}

We randomized the data into two groups of random sizes of about 312 patients at a ratio of 7:3 to develop nomograms. Detailed clinical characteristics of patients included in the training cohort and 
internal validation cohort are shown in Table 2. There were no significant differences in the clinical characteristics between these two cohorts. In multivariate analysis, patient age at diagnosis, nuclear grade, $\mathrm{PR}$, and $\mathrm{Ki}-67$ were all found to be independent predictors of MMP low risk in this population. The odds ratio and coefficient associated with four significant factors in the multivariate model are shown in Table 3. A strong PR status was a positive effect, and a higher nuclear grade, younger age, and a higher $\mathrm{Ki}-67$ were negative effects in the MMP low-risk group. The significant variables of the explanatory model were used to develop a nomogram to predict low risk of MMP (Fig. 1). Values lower than 0.1 and higher or equal to 0.9 were not displayed. The total scores for individual patients ranged from 81 to 189 . The overall predictive accuracy of the nomogram was measured based on the AUC, which was 0.82 (95\% confidence interval [Cl], 0.77 to 0.87$)$ for the training dataset of 312 patients, and $0.77(95 \% \mathrm{Cl}, 0.68$ to 0.86 ) for the internal validation dataset of 97 patients (Fig. 2).

Sensitivity, specificity and positive and negative predictive values

Table 4 shows the sensitivity, specificity, and positive and negative predictive values according to each cutoff value. Each probability shown in the table represents the cutoff value of different probabilities of obtaining a low-risk MMP results; for example, when we apply a probability of $90 \%$ as a cutoff for binary risk results, it means that the nomogram results higher than $90 \%$ will have a higher chance of being associated with actual MMP low risk; the specificity, sensitivity, positive predictive value (PPV), and negative predictive value (NPV) are shown. A positive predictive value refers to the ability to predict MMP low risk without MMP testing. Based on our results, use of a low cutoff value strongly predicted the actual MMP high s risk, with 0.90 (38/42) PPV and $98 \%$ sensitivity. With a high cutoff value of $90 \%$, we predicted an actual low-risk MMP result with an NPV of $0.89(4 / 34)$ and a $98 \%$ specificity. The specificity rate was also determined to investigate the clinical utility of the nomogram.

\section{User-friendly calculator}

The results obtained from our analysis were used to develop a user-friendly calculator using Microsoft Excel worksheets (Fig. 3). The interface allows the user to input the values of age at diagnosis, PR Allred score (0-8), Ki-67 level as a percentage, and nuclear grade (1-3). The standard output includes an estimate of the probability of detecting low MMP risk when actually tested. Our algorithm seems to help clinicians in identifying patients with a higher chance of getting a low risk or high risk MMP test and for whom gene testing accessibility is low.

\section{Discussion}

MMP is the one of the most widely used genomic assays for breast cancer testing in the world, especially for patients with one to three positive nodes. The recent National Comprehensive Cancer Network (NCCN) guideline recommends clinicians to consider MMP testing for decision making regarding adjuvant systemic chemotherapy with evidence of category 1, for patients with ER/PR positive, HER2 negative, and with negative or positive (1-3) node metastasis breast cancer [8]. Although according to interim analysis of RxPONDER trial, the guideline recommends Oncotype DX for same subset of patients, however the 
interpretation of the results in premenopausal patients in complex. [9] The use of genomic signatures is recommended for this subset of patients by national and international clinical guidelines, i.e., St. Gallen Consensus Conference, European Society for Medical Oncology (ESMO), and American Society of Clinical Oncology [13-15]. However the economic burden on patients makes clinicians hesitate to recommend genomic testing. Although reports on the cost effectiveness of MMP show that MMP safely guided chemotherapy de-escalation in clinical high-risk patients with HR+/HER2- tumors compared (compared to clinical assessment alone) [16], the cost of MMP in South Korea (approximately $\$ 3,200$ ) can still be a burden for patients. As this test originally designed in foreign countries, the South Korea National Health Insurance does not cover the test, only a few private health insurance companies covers MMP. Therefore, MMP imposes an economic burden in South Korea, regardless of whether patients have private insurance or not. This study was performed to evaluate MMP risk assessment, which was based on routine standard patient and tumor characteristics. Similar prediction models have been developed using clinicopathological data [17] or radiological phenotype results [18]. However, the best way to link these results with clinical practice has not yet been identified. We found that using this prediction tool with simple four combined clinicopathological factors can aid decision-making and serve as an alternative to the costly MMP test for patients by predicting high- or low-risk MMP results.

Four clinicopathological variables were used in our model, age, nuclear grade, PR, and Ki-67. The values for the latter three variables can be easily determined through examination in any pathological laboratory in health care centers and were used to predict prognosis before the gene testing era. Here, the Allred score of ER was not included in the final model because most of the patients had high (7-8) ER scores with variation in the PR status only. This implies that clinicians have a tendency to perform fewer genomic tests to decide on adjuvant chemotherapy administration for patients with weak to intermediate ER scores (3-6) $[19,20]$. A higher ER status is related to a higher endocrine response and a lower chemotherapy response [21]. In contrast, a low ER status is known to be associated with a low chemotherapy response, which is similar to negative ER tumors, compared with strong ER-positive tumors in neoadjuvant settings [22]. PR status can also be used to predict the endocrine and chemotherapy responses. By analyzing 77 invasive breast cancers and their PR status and 21-gene testing recurrence score results, a strong negative correlation between both factors was revealed [20].

Another important clinical factor according to our model was the age at diagnosis. A negative association was observed between younger patients and a high nomogram score. The chemotherapy benefit for invasive disease-free survival varied when the recurrence score was combined with age $(p=$ 0.004), with some chemotherapy benefits found in women $<50$ years with a recurrence score of $16-25$ [5]. According to a recent update on the long-term results of MINDACT trial (EORTC 10041/BIG3-04) presented at the American society of clinical oncology annual meeting in 2020, there is an absolute $5 \% \pm$ $2.8 \%$ distant metastasis free survival gain with adjuvant chemotherapy in premenopausal women with a discordant clinical and genomic risk (clinical high risk/genomic low risk) [11]. The authors suggested that this result is due to chemotherapy-induced ovarian function suppression. In a recent phase III trial of 1,483 premenopausal women with ER-positive breast cancer with neoadjuvant or adjuvant chemotherapy, patients who recovered their ovarian function after chemotherapy showed a better overall survival upon 
adding ovarian function suppression with tamoxifen (compared with the tamoxifen-alone group) [23]. According to our prediction model, age was the second most powerful predictor of MMP risk results. Younger patients had a higher probability of receiving MMP high-risk results. These results should be interpreted with caution as even the same genomic scores can differ in their long-term outcome based on age. The recent analysis of MINDACT trial revealed that there was no significant difference in the longterm outcome between clinical-low/genomic-low risk and clinical-low/genomic-high risk groups. When applied to our model, if the clinicopathological indicators other than age are positively correlated with a low risk, endocrine and ovarian function suppression would be a better option than MMP or chemotherapy.

In our previous study on a prediction model for Oncotype DX recurrence score [12], Ki-67 was most strongly related to the Recurrence Score. The role of Ki-67 as an indicator of poor prognosis in the Oncotype Dx gene assay is well-known [24-27]. Similarly, a current study also revealed that a higher Ki67 level was closely associated with MMP high risk. Ki-67 itself can be a strong prognostic index; however, recent analyses on intermediate Ki-67 and MMP results showed that for the patients with a low Ki-67 $(<15 \%)$ or a high Ki-67 ( $>30 \%)$, the risk results of the MMP test mostly agreed with the Ki-67 level, while for the patients with an intermediate Ki-67 value (15-30\%), they were discordant with the MMP risk result [28]. It is expected that with a definitely high $\mathrm{Ki}-67$, clinicians would be reluctant to forego adjuvant chemotherapy or make a decision after MMP testing. When we looked up our data closely, in $175 \mathrm{Ki}-67$ high ( $\geq 20 \%$ ) patients, 135 patients (77.1\%) had an intermediate Ki-67 level (20-40) and only 40 patients $(22.8 \%)$ had a Ki-67 level $>40$. Our prediction model would give clinicians a better decision guide for a low to intermediate Ki-67 level, combined with the other factors. Grade has also been long regarded as a prognostic indicator of breast cancer outcome [29] by Nottingham Prognostic Index, whose association with genomic assays is proven [30].

This study has some strength. The majority of the patients were $(94.8 \%)$ node positive, with up to three lymph nodes. The final results of the lymph-node-positive population with an Oncotype DX recurrence score (RxPONDER) [31] are pending, MMP is the only recommended genomic assay, in accordance with evidence category 1 in NCCN guideline regardless of menopausal status. We also analyzed age, which plays a key role in interpreting two major genomic tests according to recent publications $[5,11]$, making the decision to de-escalate treatment by adding ovarian suppression to conventional endocrine therapyinstead of chemotherapy-making it much easier for clinicians who hesitate to test premenopausal patients who can hardly afford the genomic testing. The MINDACT trial for MMP mainly comprised Caucasians [32], requiring the acquisition of more data by doing a retrospective analysis on Asian populations. Our nomogram can provide additional information to clinicians in Asian countries who are planning to use genomic assays to de-escalate or escalate adjuvant therapy. We also offer a user-friendly interfaced calculator with simple four robust factors which can be widely used by clinicians. This study has also some limitations. Due to its retrospective nature, our study might have selection bias with respect to the nature of the primary population, i.e., patients with a low MMP risk. However, this is also a strength, as this population reflects a subset about which clinicians ponder for performing MMP testing. Also, there is a concern that reproducibility of Ki-67 level due to variability of the assay which has not 
been validated by the St. Gallen guidelines. Because a prediction nomogram will never produce the same result as a genomic test, we suggest that the purpose of using this nomogram should be to decide on whether to perform genomic test for intermediate risk patients who cannot afford the associated medical expense. Further, the study endpoint was set based on the result of MMP, not the subsequent outcome of the patient. we recommend that clinicians interpret and apply the nomogram results carefully after sufficient agreement with patients.

In conclusion, our nomogram, which predicts a low-risk MMP result, will be a useful tool to help select patients with HR+/HER2- and node-positive tumors who may or may not need additional MMP testing, especially with intermediate clinicopathological characteristics. It may also be a useful replacement for MMP testing in cases where genomic testing can be costly or when MMP testing is not available.

\section{Declarations}

\section{Acknowledgement}

We would like to thank Editage (www.editage.co.kr) for English language editing.

\section{Authors' contributions}

Conception or design of the work: Young Joo Lee, Junetae Kim and Sae Byul Lee

Data collection: Young Joo Lee, Sei-Hyun Ahn, Byung Ho Son, Hee Jeong Kim, Beom Seok Ko, Jisun Kim, II Yong Chung, Jong Won Lee and Sae Byul Lee

Data analysis and interpretation: Young Joo Lee, Young Sol Hwang, Junetae Kim and Sae Byul Lee

Drafting the article: Young Joo Lee and Sae Byul Lee

Critical revision of the article: Sae Byul Lee

Final approval of the version to be published: All authors

\section{Funding}

This study was supported by a grant (grant number 2020-0037) from the Asan Institute for Life Sciences, Asan Medical Center, Seoul, Korea.

\section{Conflicts of interest/Competing interests}

The authors declare that there is no conflict of interest.

\section{Ethics approval}


The project was reviewed and approved by the Asan Medical Center institutional review board (20200037). Due to the retrospective nature of the study, the requirement for informed consent was waived.

\section{Consent to participate}

Not applicable

\section{Consent for publication}

Not applicable

\section{Availability of data and material}

Data archiving is not mandated but data will be made available on reasonable request

\section{Code availability}

Not applicable

\section{References}

1. Rosenberg, J., Chia, Y. L. \& Plevritis, S. The effect of age, race, tumor size, tumor grade, and disease stage on invasive ductal breast cancer survival in the US SEER database. Breast Cancer Res. Treat. 89, 47-54 (2005).

2. Koscielny, S. et al. Breast cancer: relationship between the size of the primary tumour and the probability of metastatic dissemination. Br. J. Cancer 49, 709-715 (1984).

3. Fisher, B. et al. Relation of number of positive axillary nodes to the prognosis of patients with primary breast cancer. An NSABP update. Cancer 52, 1551-1557 (1983).

4. Baak, J. P. et al. Proliferation is the strongest prognosticator in node-negative breast cancer: significance, error sources, alternatives and comparison with molecular prognostic markers. Breast Cancer Res. Treat. 115, 241-254 (2009).

5. Sparano, J. A. et al. Adjuvant chemotherapy guided by a 21-gene expression assay in breast cancer. N. Engl. J. Med. 379, 111-121 (2018).

6. Dowsett, M. et al. Prediction of risk of distant recurrence using the 21-gene recurrence score in nodenegative and node-positive postmenopausal patients with breast cancer treated with anastrozole or tamoxifen: a TransATAC study. J. Clin. Oncol. 28, 1829-1834 (2010).

7. Mamounas, E. P. et al. Association between the 21-gene recurrence score assay and risk of locoregional recurrence in node-negative, estrogen receptor-positive breast cancer: results from NSABP B-14 and NSABP B-20. J. Clin. Oncol. 28, 1677-1683 (2010).

8. Cardoso, F. et al. 70-gene signature as an aid to treatment decisions in early-stage breast cancer. $N$. Engl. J. Med. 375, 717-729 (2016). 
9. Trials.gov, C. Tamoxifen citrate, letrozole, anastrozole, or exemestane With or Without chemotherapy in treating patients with invasive RxPONDER breast cancer. https://clinicaltrials.gov/ct2/show/NCT01272037 (2011).

10.

11. Cardoso, F. et al. MINDACT: long-term results of the large prospective trial testing the 70-gene signature MammaPrint as guidance for adjuvant chemotherapy in breast cancer patients (American Society of Clinical Oncology, 2020).

12. Lee, S. B. et al. A nomogram for predicting the Oncotype DX recurrence score in women with T1-3NO1 miM0 hormone receptor-positive, human epidermal growth factor 2 (HER2)-negative breast cancer. Cancer Res. Treat. 51, 1073-1085 (2019).

13. Krop, I. et al. Use of biomarkers to guide decisions on adjuvant systemic therapy for women with early-stage invasive breast cancer: American Society of Clinical Oncology clinical practice guideline focused update. J. Clin. Oncol. 35, 2838 (2017).

14. Morigi, C. Highlights from the 15th st Gallen International Breast Cancer Conference 15-18 March, 2017, Vienna: tailored treatments for patients with early breast cancer. Ecancermedicalscience 11, 732 (2017).

15. Senkus, E. et al. Primary breast cancer: ESMO Clinical Practice Guidelines for diagnosis, treatment and follow-up. Ann. Oncol. 26, v8-v30 (2015).

16. Retèl, V. P. et al. Cost-effectiveness analysis of the 70-gene signature compared with clinical assessment in breast cancer based on a randomised controlled trial. Eur. J. Cancer 137, 193-203 (2020).

17. Dabbs, D. et al. Abstract P5-07-04: Pathology data predicts MammaPrint result-The Magee MammaPrint equation (American Association for Cancer Research, 2016).

18. $\mathrm{Li}, \mathrm{H}$. et al. MR imaging radiomics signatures for predicting the risk of breast cancer recurrence as given by research versions of MammaPrint, Oncotype DX, and PAM50 gene assays. Radiology 281, 382-391 (2016).

19. Paik, S. et al. Gene expression and benefit of chemotherapy in women with node-negative, estrogen receptor-positive breast cancer. J. Clin. Oncol. 24, 3726-3734 (2006).

20. Tang, P. et al. A lower Allred score for progesterone receptor is strongly associated with a higher recurrence score of 21-gene assay in breast cancer. Cancer Invest. 28, 978-982 (2010).

21. Lippman, M. E. \& Allegra, J. C. Quantitative estrogen receptor analyses: the response to endocrine and cytotoxic chemotherapy in human breast cancer and the disease-free interval. Cancer 46, 28292834 (1980).

22. Landmann, A. et al. Low estrogen receptor (ER)-positive breast cancer and neoadjuvant systemic chemotherapy: is response similar to typical ER-positive or ER-negative disease? Am. J. Clin. Pathol. 150, 34-42 (2018).

23. Kim, H. A. et al. Adding ovarian suppression to tamoxifen for premenopausal breast cancer: A randomized phase III trial. J. Clin. Oncol. 38, 434-443 (2020). 
24. Williams, D. J., Cohen, C., Darrow, M., Page, A. J., Chastain, B. \& Adams, A. L. Proliferation (Ki-67 and phosphohistone $\mathrm{H} 3$ ) and oncotype $\mathrm{DX}$ recurrence score in estrogen receptor-positive breast cancer. Appl. Immunohistochem. Mol. Morphol. 19, 431-436 (2011).

25. Sahebjam, S. et al. Ki 67 is a major, but not the sole determinant of Oncotype Dx recurrence score. $B r$. J. Cancer 105, 1342-1345 (2011).

26. Nishimura, R., Osako, T., Okumura, Y., Hayashi, M. \& Arima, N. Clinical significance of Ki-67 in neoadjuvant chemotherapy for primary breast cancer as a predictor for chemosensitivity and for prognosis. Breast Cancer 17, 269-275 (2010).

27. Viale, G. et al. Prognostic and predictive value of centrally reviewed Ki-67 labeling index in postmenopausal women with endocrine-responsive breast cancer: results from Breast International Group Trial 1-98 comparing adjuvant tamoxifen with letrozole. J. Clin. Oncol. 26, 5569-5575 (2008).

28. Tian, C. et al. Ki-67 versus MammaPrint/BluePrint for assessing luminal type breast cancer (American Society of Clinical Oncology, 2020).

29. Galea, M. H., Blamey, R. W., Elston, C. E. \& Ellis, I. O. The Nottingham Prognostic Index in primary breast cancer. Breast Cancer Res. Treat. 22, 207-219 (1992).

30. Carlson, J. J. \& Roth, J. A. The impact of the Oncotype Dx breast cancer assay in clinical practice: a systematic review and meta-analysis. Breast Cancer Res. Treat. 141, 13-22 (2013).

31. Jasem, J. et al. The 21-gene recurrence score assay for node-positive, early-stage breast cancer and impact of RXPONDER Trial on chemotherapy decision-making: have clinicians already decided? J. Natl Compr. Canc. Netw. 15, 494-503 (2017).

32. Cardoso, F., Piccart-Gebhart, M., Van't Veer, L., Rutgers, E. \& TRANSBIG Consortium. The MINDACT trial: the first prospective clinical validation of a genomic tool. Mol. Oncol. 1, 246-251 (2007).

\section{Tables}


Table 1

Baseline characteristics of the patient cohort

\begin{tabular}{|c|c|c|c|}
\hline \multirow[t]{2}{*}{ Variables } & \multirow{2}{*}{$\begin{array}{l}\text { MMPa low-risk } \\
(\mathrm{N}=240)\end{array}$} & \multirow{2}{*}{$\begin{array}{l}\text { MMPa high-risk } \\
(\mathrm{N}=169)\end{array}$} & \multirow[t]{2}{*}{ p-value } \\
\hline & & & \\
\hline & $\mathrm{N}(\%)$ & $\mathrm{N}(\%)$ & \\
\hline Age at diagnosis $\left(\right.$ mean $\left.\pm \mathrm{SD}^{\star}\right)$ & $53.3 \pm 9.3$ & $47.9 \pm 9.8$ & $<0.001$ \\
\hline Histological grade & & & $<0.001$ \\
\hline - grade I & $19(8.0 \%)$ & $3(1.8 \%)$ & \\
\hline - grade II & $218(90.8 \%)$ & $139(82.2 \%)$ & \\
\hline - grade III & $3(1.2 \%)$ & $27(16.0 \%)$ & \\
\hline Nuclear grade & & & $<0.001$ \\
\hline - grade I & $2(0.8 \%)$ & $0(0.0 \%)$ & \\
\hline - grade II & $235(97.9 \%)$ & $140(82.8 \%)$ & \\
\hline - grade III & $3(1.3 \%)$ & $29(17.2 \%)$ & \\
\hline Estrogen receptor & & & $<0.001$ \\
\hline - negative & $0(0.0 \%)$ & $0(0.0 \%)$ & \\
\hline - weak & $0(0.0 \%)$ & $0(0.0 \%)$ & \\
\hline - intermediate & $2(0.8 \%)$ & $6(3.6 \%)$ & \\
\hline - strong & $238(99.2 \%)$ & $163(96.4 \%)$ & \\
\hline Progesterone receptor & & & $<0.001$ \\
\hline - negative & $16(6.6 \%)$ & $21(12.4 \%)$ & \\
\hline - weak & $12(5.0 \%)$ & $25(14.8 \%)$ & \\
\hline - intermediate & $47(19.6 \%)$ & $33(19.5 \%)$ & \\
\hline - strong & $165(68.8 \%)$ & $90(53.3 \%)$ & \\
\hline Lymphovascular invasion & & & $<0.001$ \\
\hline - negative & $147(61.2 \%)$ & $67(40.4 \%)$ & \\
\hline - positive & $93(38.8 \%)$ & $99(59.6 \%)$ & \\
\hline $\mathrm{p}-53$ & & & $<0.001$ \\
\hline-0 & $78(32.5 \%)$ & $56(33.1 \%)$ & \\
\hline
\end{tabular}




\begin{tabular}{|c|c|c|c|}
\hline \multirow[t]{2}{*}{ Variables } & MMPa low-risk & MMPa high-risk & \multirow[t]{2}{*}{ p-value } \\
\hline & $(\mathrm{N}=240)$ & $(N=169)$ & \\
\hline-1 & $109(45.4 \%)$ & $51(30.2 \%)$ & \\
\hline-2 & $43(17.9 \%)$ & $33(19.5 \%)$ & \\
\hline-3 & $10(4.2 \%)$ & $29(17.2 \%)$ & \\
\hline Ki-67 level & & & $<0.001$ \\
\hline - low Ki-67<20\% & $177(73.8 \%)$ & $57(33.7 \%)$ & \\
\hline - high Ki-67 $\geq 20 \%$ & $63(26.2 \%)$ & $112(66.3 \%)$ & \\
\hline Breast surgery & & & 0.675 \\
\hline - total mastectomy & $69(28.8 \%)$ & $44(26.3 \%)$ & \\
\hline - breast conservation surgery & $171(71.2 \%)$ & $123(73.7 \%)$ & \\
\hline Axillary operation & & & 0.056 \\
\hline & $N(\%)$ & $\mathrm{N}(\%)$ & \\
\hline - axillary dissection & $3(1.3 \%)$ & $0(0.0 \%)$ & \\
\hline - sentinel node biopsy & $133(55.6 \%)$ & $78(46.7 \%)$ & \\
\hline - axillary dissection after sentinel node biopsy & $103(43.1 \%)$ & $89(53.3 \%)$ & \\
\hline T stage & & & 0.045 \\
\hline$-\mathrm{T} 1$ & $124(51.7 \%)$ & $67(40.1 \%)$ & \\
\hline$-\mathrm{T} 2$ & $111(46.2 \%)$ & $98(58.7 \%)$ & \\
\hline$-\mathrm{T3}$ & $5(2.1 \%)$ & $2(1.2 \%)$ & \\
\hline $\mathrm{N}$ stage & & & 0.416 \\
\hline - NO & $9(3.8 \%)$ & $10(6.0 \%)$ & \\
\hline - N1 & $231(96.2 \%)$ & $157(94.0 \%)$ & \\
\hline Stage & & & 0.449 \\
\hline - Stage I & $31(12.9 \%)$ & $16(9.6 \%)$ & \\
\hline - Stage II & $204(85.0 \%)$ & $149(89.2 \%)$ & \\
\hline - Stage III & $5(2.1 \%)$ & $2(1.2 \%)$ & \\
\hline Tumor size $(\mathrm{cm})\left(\right.$ mean $\left.\pm S D^{\star}\right)$ & $2.2 \pm 1.1$ & $2.4 \pm 1.0$ & 0.018 \\
\hline Number of positive nodes & & & 0.942 \\
\hline
\end{tabular}




\begin{tabular}{|c|c|c|c|}
\hline \multirow[t]{2}{*}{ Variables } & \multirow{2}{*}{$\begin{array}{l}\text { MMPa low-risk }^{\mathrm{a}} \\
(\mathrm{N}=240)\end{array}$} & \multirow{2}{*}{$\begin{array}{l}\text { MMPa high-risk } \\
(\mathrm{N}=169)\end{array}$} & \multirow[t]{2}{*}{ p-value } \\
\hline & & & \\
\hline-0 & $9(3.8 \%)$ & $8(4.8 \%)$ & \\
\hline-1 & $150(62.5 \%)$ & $105(62.9 \%)$ & \\
\hline-2 & $66(27.5 \%)$ & $45(26.9 \%)$ & \\
\hline-3 & $15(6.2 \%)$ & $9(5.4 \%)$ & \\
\hline Largest positive node size (mm) & $5.4 \pm 3.9$ & $6.3 \pm 4.8$ & 0.042 \\
\hline Menopausal status & & & $<0.001$ \\
\hline - premenopause & $112(46.7 \%)$ & $113(66.9 \%)$ & \\
\hline - postmenopause & $128(53.3 \%)$ & $55(32.5 \%)$ & \\
\hline - unknown & $0(0.0 \%)$ & $1(0.6 \%)$ & \\
\hline
\end{tabular}


Table 2

Characteristics of Validation and Training groups.

\begin{tabular}{|c|c|c|c|}
\hline \multirow[t]{2}{*}{ Variables } & \multirow{2}{*}{$\begin{array}{l}\text { Validation set } \\
(\mathrm{N}=97)\end{array}$} & \multirow{2}{*}{$\begin{array}{l}\text { Training set } \\
(\mathrm{N}=312)\end{array}$} & \multirow[t]{2}{*}{$p$-value } \\
\hline & & & \\
\hline & $\mathrm{N}(\%)$ & $\mathrm{N}(\%)$ & \\
\hline Age at diagnosis (mean $\pm \mathrm{SD}^{\star}$ ) & $51.1 \pm 10.3$ & $51.1 \pm 9.8$ & 0.989 \\
\hline MammaPrint results & & & 0.297 \\
\hline - low risk & $52(53.6 \%)$ & $188(60.3 \%)$ & \\
\hline - high risk & $45(46.4 \%)$ & $124(39.7 \%)$ & \\
\hline Histological grade & & & 0.818 \\
\hline - grade I & $4(4.1 \%)$ & $18(5.8 \%)$ & \\
\hline - grade II & $86(88.7 \%)$ & $271(86.9 \%)$ & \\
\hline - grade III & $7(7.2 \%)$ & $23(7.3 \%)$ & \\
\hline Nuclear grade & & & 0.662 \\
\hline - grade I & $1(1.0 \%)$ & $1(0.3 \%)$ & \\
\hline - grade II & $89(91.8 \%)$ & $286(91.7 \%)$ & \\
\hline - grade III & $7(7.2 \%)$ & $25(8.0 \%)$ & \\
\hline Estrogen receptor & & & 0.686 \\
\hline - negative & $0(0.0 \%)$ & $0(0.0 \%)$ & \\
\hline - weak & $0(0.0 \%)$ & $0(0.0 \%)$ & \\
\hline - intermediate & $1(1.0 \%)$ & $7(2.2 \%)$ & \\
\hline - strong & $96(99.0 \%)$ & $305(97.8 \%)$ & \\
\hline Progesterone receptor & & & 0.072 \\
\hline - negative & $15(15.5 \%)$ & $22(7.1 \%)$ & \\
\hline - weak & $6(6.2 \%)$ & $31(9.9 \%)$ & \\
\hline - intermediate & $20(20.6 \%)$ & $60(19.2 \%)$ & \\
\hline - strong & $56(57.7 \%)$ & $199(63.8 \%)$ & \\
\hline Lymphovascular invasion & & & 0.797 \\
\hline - negative & $49(51.0 \%)$ & $165(53.2 \%)$ & \\
\hline
\end{tabular}




\begin{tabular}{|c|c|c|c|}
\hline \multirow[t]{2}{*}{ Variables } & Validation set & Training set & \multirow[t]{2}{*}{$p$-value } \\
\hline & $(\mathrm{N}=97)$ & $(N=312)$ & \\
\hline - positive & $47(49.0 \%)$ & $145(46.8 \%)$ & \\
\hline p-53 & & & 0.289 \\
\hline-0 & $37(38.1 \%)$ & $97(31.1 \%)$ & \\
\hline-1 & $38(39.2 \%)$ & $122(39.1 \%)$ & \\
\hline-2 & $17(17.5 \%)$ & $59(18.9 \%)$ & \\
\hline-3 & $5(5.2 \%)$ & $34(10.9 \%)$ & \\
\hline Ki-67 level & & & 0.999 \\
\hline - low Ki-67<20\% & $56(57.7 \%)$ & $178(57.1 \%)$ & \\
\hline - high Ki-67 $\geq 20 \%$ & $41(42.3 \%)$ & $134(42.9 \%)$ & \\
\hline Breast surgery & & & 0.528 \\
\hline - total mastectomy & $24(24.7 \%)$ & $89(28.7 \%)$ & \\
\hline - breast conservation surgery & $73(75.3 \%)$ & $221(71.3 \%)$ & \\
\hline Axillary operation & & & 0.927 \\
\hline - axillary dissection & $1(1.0 \%)$ & $2(0.6 \%)$ & \\
\hline - sentinel node biopsy & $50(51.5 \%)$ & $161(52.1 \%)$ & \\
\hline - axillary dissection after sentinel node biopsy & $46(47.5 \%)$ & $146(47.3 \%)$ & \\
\hline T stage & & & 0.836 \\
\hline$-\mathrm{T} 1$ & $46(47.4 \%)$ & $145(46.8 \%)$ & \\
\hline$-\mathrm{T} 2$ & $50(51.5 \%)$ & 159 (51.3\%) & \\
\hline - T3 & $1(1.1 \%)$ & $6(1.9 \%)$ & \\
\hline $\mathrm{N}$ stage & & & 0.571 \\
\hline - NO & $3(3.1 \%)$ & $16(5.2 \%)$ & \\
\hline - N1 & 94 (96.9\%) & $294(94.8 \%)$ & \\
\hline Stage & & & 0.808 \\
\hline - Stage I & $12(12.4 \%)$ & $35(11.3 \%)$ & \\
\hline - Stage II & $84(86.6 \%)$ & $269(86.8 \%)$ & \\
\hline - Stage III & $1(1.0 \%)$ & $6(1.9 \%)$ & \\
\hline
\end{tabular}




\begin{tabular}{|c|c|c|c|}
\hline \multirow[t]{2}{*}{ Variables } & Validation set & Training set & \multirow[t]{2}{*}{$p$-value } \\
\hline & $(N=97)$ & $(N=312)$ & \\
\hline Tumor size $(\mathrm{cm})\left(\right.$ mean $\left.\pm S D^{\star}\right)$ & $2.2 \pm 1.1$ & $2.3 \pm 1.1$ & 0.678 \\
\hline \multicolumn{3}{|l|}{ Number of positive nodes } & \multirow[t]{5}{*}{0.340} \\
\hline-0 & $1(1.0 \%)$ & $16(5.2 \%)$ & \\
\hline-1 & $61(62.9 \%)$ & $194(62.5 \%)$ & \\
\hline-2 & $29(29.9 \%)$ & $82(26.5 \%)$ & \\
\hline-3 & $6(6.2 \%)$ & $18(5.8 \%)$ & \\
\hline Largest positive node size (mm) & $6.4 \pm 4.9$ & $5.6 \pm 4.1$ & 0.112 \\
\hline \multicolumn{3}{|l|}{ Menopausal status } & \multirow[t]{4}{*}{0.850} \\
\hline - premenopause & $53(54.6 \%)$ & $172(55.1 \%)$ & \\
\hline - postmenopause & $44(45.4 \%)$ & $139(44.6 \%)$ & \\
\hline - unknown & $0(0.0 \%)$ & $1(0.3 \%)$ & \\
\hline
\end{tabular}

Table 3

Multivariate logistic regression model

\begin{tabular}{|c|c|c|c|c|c|}
\hline \multirow[t]{2}{*}{ Variables } & \multicolumn{5}{|c|}{ Multivariate model } \\
\hline & $\begin{array}{l}\beta \\
\text { coefficient }\end{array}$ & $\begin{array}{l}\text { Standard } \\
\text { error }\end{array}$ & $\begin{array}{l}Z \\
\text { score }\end{array}$ & $p$-value & $\begin{array}{l}95 \% \text { confidence } \\
\text { interval }\end{array}$ \\
\hline Age & 0.653554 & 0.016 & 3.92 & 0.000 & $0.032-0.098$ \\
\hline $\begin{array}{l}\text { Progesterone receptor } \\
\text { status }\end{array}$ & 0.1812972 & 0.780 & -5.61 & 0.005 & $0.054-0.307$ \\
\hline Nuclear grade & -2.223743 & 0.064 & -2.85 & 0.004 & $-3.754-0.693$ \\
\hline Ki-67 & -0.0686273 & 0.012 & 1.06 & 0.000 & $-1.687--0.044$ \\
\hline
\end{tabular}


Table 4

Sensitivity, specificity, positive predictive, and negative predictive values according to various cutoff values

\begin{tabular}{|c|c|c|c|c|c|c|}
\hline $\begin{array}{l}\text { Probability } \\
(\%)\end{array}$ & $\begin{array}{l}\text { Low risk of MMP } \\
(\mathrm{N})\end{array}$ & $\begin{array}{l}\text { High risk of MMP } \\
(\mathrm{N})\end{array}$ & $\begin{array}{l}\text { Sensitivity } \\
\text { (\%) }\end{array}$ & $\begin{array}{l}\text { Specificity } \\
\text { (\%) }\end{array}$ & $\begin{array}{l}\text { PPVa }^{a} \\
\text { (\%) }\end{array}$ & $\begin{array}{l}\text { NPVb } \\
\text { (\%) }\end{array}$ \\
\hline \multirow[t]{2}{*}{10} & 236 & 131 & 98 & 22 & 90 & 64 \\
\hline & 4 & 38 & & & & \\
\hline \multirow[t]{2}{*}{35} & 229 & 99 & 95 & 46 & 86 & 70 \\
\hline & 11 & 70 & & & & \\
\hline \multirow[t]{2}{*}{55} & 206 & 61 & 86 & 64 & 76 & 77 \\
\hline & 34 & 108 & & & & \\
\hline \multirow[t]{2}{*}{80} & 109 & 18 & 45 & 89 & 54 & 86 \\
\hline & 131 & 151 & & & & \\
\hline \multirow[t]{2}{*}{87} & 59 & 9 & 25 & 95 & 47 & 87 \\
\hline & 181 & 160 & & & & \\
\hline \multirow[t]{2}{*}{90} & 34 & 4 & 14 & 98 & 44 & 89 \\
\hline & 206 & 165 & & & & \\
\hline
\end{tabular}

\section{Figures}


Points

$\begin{array}{llllllllll}0 & 10 & 20 & 30 & 40 & 50 & 60 & 70 & 80 & 90\end{array}$

Age

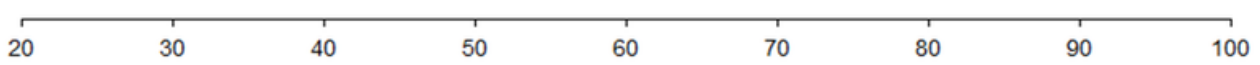

Nuclear grade

2

Progesterone

receptor

\begin{tabular}{lllll} 
& 3 & 5 & 7 \\
\hline & 1 & & 1 & \\
\hline & 4 & 6 & 8
\end{tabular}

Ki-67 (\%)

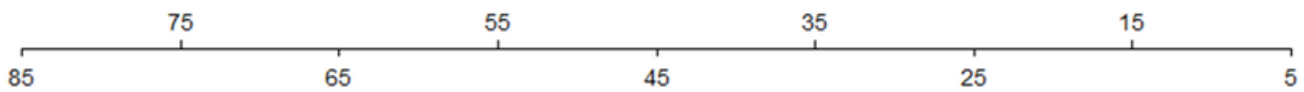

Total points

\begin{tabular}{lllllllllllllllll}
\hline 0 & 20 & 40 & 60 & 80 & 100 & 120 & 140 & 160 & 180 & 200 & 220 & 240 & 260
\end{tabular}

Low risk

\begin{tabular}{llllllll}
\hline & 0.1 & 0.2 & 0.3 & 0.40 .50 .6 & 0.7 & 0.8 & 0.9
\end{tabular}

\section{Figure 1}

Nomogram to predict a MammaPrint low risk. Age, Progesterone receptor, nuclear grade, and Ki-67 levels were finally selected to develop the model
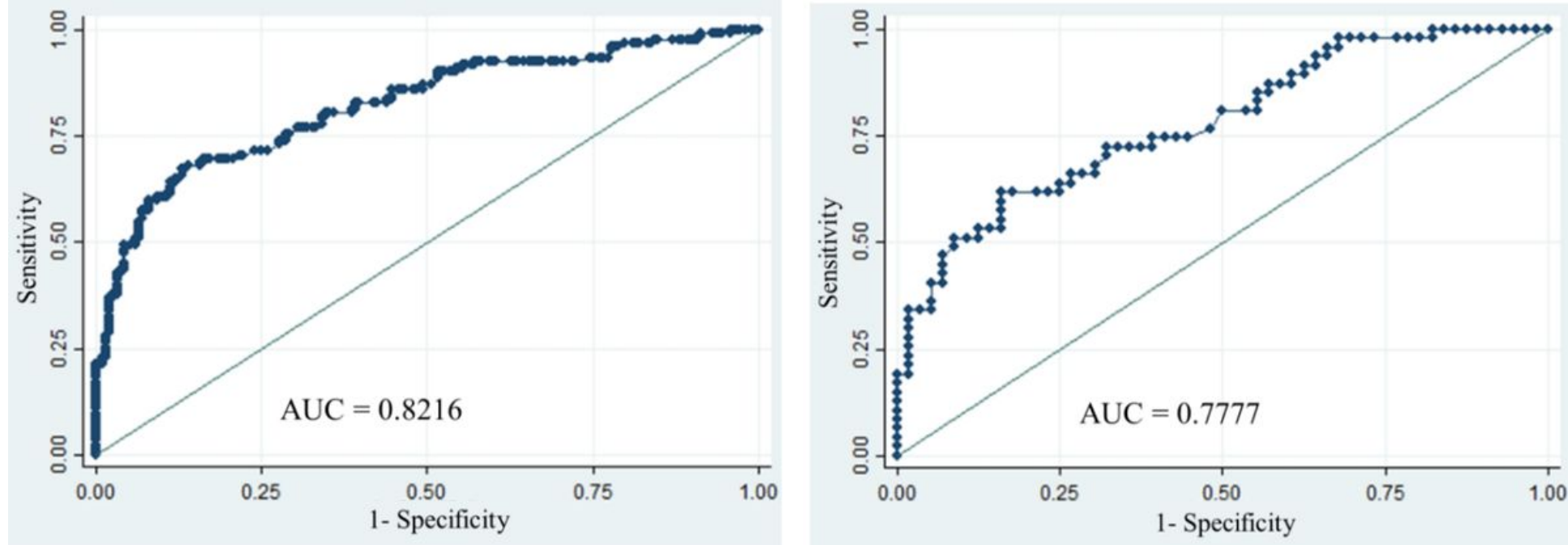
Figure 2

Receiver operating characteristic curve of nomogram. (a) Training group of 312 patients. (b) Validation group of 97 patients

\begin{tabular}{|l|c|r|}
\hline & A & \multicolumn{2}{|c|}{ B } \\
\hline 1 & & Input Value \\
\hline 2 & Age & 47 \\
\hline 3 & PgR & 7 \\
\hline 4 & Ki67 & 5 \\
\hline 5 & Nuclear & \\
\hline 6 & Constant & \\
\hline 7 & Sum & \\
\hline 8 & Risk Probability & 0.8229106 \\
\hline
\end{tabular}

Figure 3

Automatic calculator using Microsoft Excel worksheets

\section{Supplementary Files}

This is a list of supplementary files associated with this preprint. Click to download.

- calculator.xlsx 